\title{
ÉTICA Y PSICOTERAPIA: \\ UNA PERSPECTIVA SOCIOCULTURAL
}

\section{ETHICS AND PSYCHOTHERAPY: A SOCIO-CULTURAL PERSPECTIVE}

\author{
Isabel Caro Gabalda
}

Universidad de Valencia. Facultad de Psicología

Departamento de Personalidad, Evaluación y Tratamientos Psicológicos. España

Cómo referenciar este artículo/How to reference this article:

Caro Gabalda, I. (2019). Ética y psicoterapia: Una perspectiva sociocultural. Revista de Psicoterapia, 30(213), 73-91. https://doi.org/10.33898/rdp.v30i113.304

\begin{abstract}
Resumen
La meta principal de este trabajo busca comprender la ética en psicoterapia desde una perspectiva sociocultural. Para conseguirla, la psicoterapia, al ser una práctica social de cura, se relacionará con los elementos socioculturales a su base. Esta perspectiva permite ofrecer a los terapeutas algunas reflexiones éticas sobre qué (y por qué) es correcto hacer, o no, en el campo de la psicoterapia. Ejemplificar y deconstruir tales elementos facilita la formación de los terapeutas, al igual que nos permite lograr la necesidad ética de pensadores más críticos. Este artículo desarrolla estas metas situando, en primer lugar, a la psicoterapia en un contexto sociocultural y ejemplificando la influencia bidireccional entre psicoterapia y sociedad. Esto se relacionará con una perspectiva ética puesto que determina qué se permite hacer a terapeutas y clientes y cómo se construyen, socialmente, las metas y los medios adecuados de la psicoterapia. En segundo lugar, el trabajo se centrará en los tres desafios éticos principales de la psicoterapia, es decir, confianza, cuidado y poder. Existe una gran relación entre estos tres elementos y no se pueden comprender ni desarrollar adecuadamente a menos que se sitúen en el contexto de una práctica socialmente construida.

Palabras clave: ética, psicoterapia, perspectiva sociocultural
\end{abstract}

\begin{abstract}
The main aim of this paper is to understand ethics in psychotherapy from a socio-cultural perspective. To fulfill this aim, psychotherapy, as a social healing practice, is related to the socio-cultural issues underlying it. This perspective could offer therapists some ethical reflections about what (and why) is appropriatel inappropriate to be done in the psychotherapy field. Exemplifying and deconstructing such issues could facilitate therapists' training, and could fulfill an ethical need for more critical thinkers. This paper works toward these aims by first placing psychotherapy in a sociocultural context and exemplifying the bidirectional influence between psychotherapy and society. This will be related to an ethics perspective because it implies what therapists and clients are entitled to do and how appropriate psychotherapy aims and means are socially constructed. Second, itfocuses on the three main ethical challenges in psychotherapy; i.e., trust, caring and power. These three issues are closely related and cannot be properly understood and developed unless they are placed in a socially constructed practice context.
\end{abstract}

Keywords: ethics, psychotherapy, socio-cultural perspective

Fecha de recepción: 3 de abril de 2019. Fecha de aceptación: 10 de mayo de 2019.

Correspondencia sobre este artículo:

E-mail: Isabel.Caro@uv.es

Dirección postal: Universidad de Valencia. Facultad de Psicología. Departamento de

Personalidad, Evaluación y Tratamientos Psicológicos. Avda. Blasco Ibáñez, 21. 46010-

Valencia (España)

(C) 2019 Revista de Psicoterapia 


\section{Introducción: Ética, valores y psicoterapia}

La ética es un elemento clave en el centro de la práctica terapéutica. Clarkson (2005) la definió como: "Aquel campo de la filosofía dedicado al estudio de lo bueno y lo malo. Se emplea, igualmente, para describir un conjunto de reglas acordado por un grupo de personas sobre la forma mejor o peor de comportarse" (p. 102).

Las guías éticas son de gran interés y se regulan por códigos diferentes en distintas culturas y contextos, ofreciendo a los terapeutas normas sobre lo que es apropiado hacer o no, o debe evitarse (Colegio Oficial de Psicólogos, COP, 2018; Gabriel, 2005; Jenkins, 2002; Koocher, 2007; Pope y Vasquez, 2011; Zur, 2007).

Todas estas guías son importantes, contribuyendo a comprender y definir en qué consiste una práctica correcta. Hay que destacar que la práctica de la psicoterapia refleja determinados supuestos éticos, en muchas ocasiones no visibles para nosotros (Tjeltveit, 2004). Al mismo tiempo si, según McLeod (2001), a los psicoterapeutas no se les enseña, sino que se los socializa en determinados enfoques terapéuticos y tipos de investigación, podemos considerar una responsabilidad ética facilitar a los futuros psicoterapeutas ciertas reflexiones sobre su profesión y sobre los elementos principales a su base. Por tanto, es muy importante que los psicoterapeutas se den cuenta de los supuestos filosóficos y teóricos implícitos en los métodos que emplean para tratar a los clientes y para estudiar la psicoterapia (Elliott, 2008; Slife y Williams, 1995).

Si asumimos que la psicoterapia es una práctica de curación social (Wampold e Imel, 2015) será importante delinear y rastrear su origen sociocultural y emplearlo para comprender lo que es correcto hacer en el contexto de la ética. Deconstruir y ejemplificar tales relaciones son las metas principales de este trabajo.

Por ejemplo, si la ética responde a la pregunta sobre lo que es bueno o equivocado hacer en un contexto psicoterapéutico específico, algunas cuestiones éticas aparecerán, de forma continua, en la interacción en sesión de los integrantes de la psicoterapia. Con ello nos referimos a qué consideran adecuado, terapeutas y clientes, para sus respectivos roles, y cuál es la base facilitadora de una práctica terapéutica conjunta.

Indudablemente y en primer lugar, la respuesta será diferente dependiendo del modelo terapéutico. Sin embargo, esta respuesta no surge de la nada. Para desarrollar la meta principal de este trabajo necesitamos relacionar dicha respuesta estando totalmente integrada en un zeigeist concreto, o en un momento sociocultural específico. La psicoterapia puede ser cualquier cosa menos apolítica (Cushman, 1992). Esto supone que dependiendo del momento sociocultural, los clientes van a demandar una conducta concreta en sus terapeutas, asumiendo, al mismo tiempo, un papel determinado. Igualmente, los terapeutas asumen un papel específico, ofreciendo a sus clientes un contexto concreto basado sobre determinados supuestos construidos. La forma en la que clientes y terapeutas resuelven su trabajo conjunto está totalmente inmersa en valores, supuestos, sentimientos, actitudes, etc, 
pertenecientes a un momento histórico en particular (Caro Gabalda, 1995a, 1999).

Los valores son un tema importante para la psicología (Prilleltensky, 1997). La mayoría de las veces, los profesionales usan su conocimiento de forma equivocada, como si la psicoterapia fuera la aplicación neutra de dicho conocimiento. En este sentido, la psicoterapia se convierte en una ciencia aplicada o en una psicotecnología, predominando un punto de vista objetivista (Woolfolk y Richardson, 2008). Esta perspectiva sobre la psicoterapia no sólo se refleja en las metas y en los medios para el cambio, sino en cómo medimos dicho cambio.

Así, la gran importancia que se da a la tendencia hacia los tratamientos empíricamente validados, una regla de oro (aunque controvertida; Consumer Reports, 1995; Elliott, 1998; Seligman, 1995) para muchos investigadores, tiene importantes conexiones con la ética. Esto es así porque se relaciona con la respuesta a si es bueno recibir terapia, en qué medida lo es y cuáles deben ser las metas en relación a estos posibles beneficios (Smith, 2009). En este contexto, la investigación en terapia tiene más que ver con fuerzas socioeconómicas y culturales que con debates académicos.

Por ejemplo, y tal y como señalaron Woolfolk y Richardson (2008), la terapia cognitivo-conductual desarrolló sus métodos como una psicotecnología, estandarizando manuales, y aplicando los tratamientos como si se estuviera utilizando un fármaco. Esto les permitió formar parte de los ensayos controlados aleatorizados (David, Cristea y Hofmann, 2018; Roth y Fonagy, 1996). Este es un simple ejemplo de cómo la psicoterapia está inmersa en una práctica guiada por los valores del mercado. Desarrollemos, "una práctica terapéutica breve cada vez más rápida y eficiente, más específica y con categorías concretas del DSM, y con resultados terapéuticos más medibles, para que podamos tecnologizar e industrializar las prácticas terapéuticas" (Cushman, 2002, pp. 103-104).

Indudablemente, no se está justificando que "todo vale". Todo lo contrario. Nos gustaría sugerir que la formación del futuro investigador no puede centrarse, exclusivamente, en métodos de investigación y en conceptualizaciones sobre salud mental, técnicas, etc., sino también en reflexiones teóricas sobre las actitudes y valores a la base de estas estrategias clínicas y de investigación. Es decir, en lo que es "correcto", o "incorrecto" hacer. Por ejemplo, que hacer terapia se puede recoger por completo en un manual; que es adecuado establecer "carreras de caballos" entre terapias; que todos los sujetos dentro de un grupo experimental, por ejemplo, cambian, y lo hacen en igual medida, etc. (Elliott, 1998; Henry, 1998; Wampold, 1997).

Por tanto, si enmarcamos la psicoterapia en un contexto conceptual y sociocultural surgirán distintas preguntas y respuestas, con una conexión muy cercana con la ética. Es decir, cada modelo, al estar emplazado históricamente, regula lo que es bueno o malo, dónde focalizarse y cómo pueden desarrollar terapeutas y clientes sus roles terapéuticos. La psicoterapia se da en un contexto sociocultural que supone diversas ideologías ocultas (Woolfolk, 1992). 


\section{Psicoterapia y contexto sociocultural}

Es difícil dar una definición general de la psicoterapia, puesto que se define mejor desde perspectivas concretas. Frank, en su definición clásica de psicoterapia, estableció que ésta se considera un tipo de influencia personal que necesita de un agente de cura, de alguien que sufre y busca ayuda de este agente y una relación de cura. Esto es, una serie de contactos, más o menos estructurados entre el que cura y el que sufre, realizados, ante todo, con palabras, actos, rituales, etc. (Frank y Frank, 1991). En cualquier caso, "una forma de retórica que se basa en métodos hermenéuticos" (Frank y Frank, 1991, p. 73).

Por consiguiente, la psicoterapia focaliza y analiza los problemas de los clientes desde enfoques terapéuticos concretos, lo que facilita, tanto al terapeuta como al cliente, interpretar y enmarcar las necesidades de éste último. Una vez que dichos problemas se comprenden y se encuadran, se aplican distintas técnicas y estrategias en el contexto de un tipo diferente de relación terapéutica. Cada modelo histórico ofrece una manera específica de comprender los distintos fenómenos clínicos y las relaciones entre ellos. Según cada zeitgeist se acordarán las metas, los procedimientos y el foco de la psicoterapia.

De forma más concreta, desde una perspectiva hermenéutica, lo esencial de todas nuestras creencias, experiencias, prácticas, o nuestra propia identidad está mediado por la cultura, el lenguaje y la auto-comprensión (Richardson, 2012).

Desde una perspectiva construccionista social, Berger y Luckmann (1966) establecieron que la sociedad era un producto humano y una realidad objetiva, siendo el ser humano un producto social. De esta manera, su análisis se puede aplicar a la terapia como un producto humano. Para estos autores, lo relevante es el aspecto conceptual de la terapia. En este sentido, y siguiendo a Berger y Luckman (1966):

"Ya que la terapia se preocupa de las desviaciones de las definiciones 'oficiales' de la realidad, debe desarrollar una maquinaria conceptual para dar cuenta de tales desviaciones y para mantener las realidades que se están desafiando. Esto requiere un conocimiento que incluya una teoría sobre la desviación, un dispositivo diagnóstico, y un sistema conceptual para la "cura de las almas" (pp. 130-131).

Y añaden más adelante,

"Cuando las teorías psicológicas logran un nivel elevado de complejidad intelectual es muy probable que las administren personal especialmente formado en dicho conocimiento..... Las teorías psicológicas sirven para legitimar los procedimientos de identidad-mantenimiento e identidadreparación establecidos en la sociedad, ofreciendo el vínculo teórico entre identidad y mundo, ya que ambos están definidos socialmente y son apropiados subjetivamente" (Berger y Luckman, 1966, p. 197).

Desde este punto de vista, podemos entender la psicoterapia asumiendo la influencia bidireccional entre psicoterapia y sociedad. Woolfolk y Murphy (2004) describieron que, en el pasado, las definiciones de los seres humanos se enmarcaban 
en conceptualizaciones teológicas, morales o políticas y, en este sentido, “... la explicación sobre la naturaleza humana recae, cada vez más, en el tipo de interpretación ofrecida por psicoterapeutas y científicos conductuales. De esta manera, la psicoterapia alcanza un impacto cultural que va más allá de su efecto inmediato en los clientes. La institución de la psicoterapia se convierte en una fuente importante de costumbres, valores y puntos de vista. Igualmente, es un indicador sensible de esas mismas costumbres, valores y puntos de vista. La relación entre psicoterapia y cultura supone una influencia recíproca. La psicoterapia absorbe y refleja la cultura a la que pertenece $y$, al mismo tiempo, deja una marca distintiva en dicha cultura. La psicoterapia influye directamente en la sociedad, a través de sus efectos en la literatura, el arte, los medios de comunicación y las numerosas instituciones sociales cuyas características básicas dependen de la psicología y la psiquiatría. La institución de la psicoterapia y de sus disciplinas subyacentes responde a las necesidades que tienen las sociedades secularizadas de tener estructuras directivas, ofreciendo marcos cognitivos, "cientificamente basados", para ordenar, racionalizar y normalizar el mundo social. El orden normativo de la psicoterapia se caracteriza por la interrelación entre hecho y valor, a veces hasta el grado de que ambos se fusionen" (Woolfolk y Murphy, 2004, p. 179).

La aparición del mindfulness (o la conciencia plena) ejemplifica esta influencia mutua. Esta tendencia implica una perspectiva psicológica y terapéutica diferente. En primer lugar, debemos reconocer que la psicología, como disciplina científica, forma parte de una tradición modernista (Gergen, 1991; Kvale, 1992) y, por tanto, la psicoterapia se puede considerar como un proyecto de la modernidad, y de tipo Occidental. Por el contrario, la tendencia hacia el mindfulness refleja la aparición de una perspectiva Oriental (Kabat-Zinn, 1990). Esta tradición ha aparecido en un mundo postmoderno, globalizado, con un punto de vista inclusivo sobre diferentes culturas. Al mismo tiempo, el sí mismo aparece como fragmentado y saturado (Gergen, 1991).

Brevemente, mientras que el sujeto Occidental intentaba controlar sus pensamientos y emociones, el sujeto "mindulf" les da la bienvenida. El sujeto implícito en este enfoque ha desarrollado sus posibilidades para vivir en el aquí y ahora, siendo auto-consciente, con conciencia plena sobre lo que está experienciando. Por ello, y siguiendo a Kabat-Zinn (1999), se produce un cambio de lo que es perjudicial a lo que es correcto para un sujeto. Desde una perspectiva modernista, el terapeuta sabe lo que está equivocado, por ejemplo, un pensamiento distorsionado o irracional. Sin embargo, desde la tradición Oriental, el poder para definir lo que es equivocado o deseable y saludable recae en los clientes. Esta tendencia hacia la conciencia plena se hereda de una sociedad que busca nuevas formas de comportarse (y, por tanto, sobre lo que es bueno, o no, hacer en terapia) basadas sobre 
tradiciones y culturas valoradas de forma nueva y diferente (como la filosofía Budista).

Lo que comenzó, por ejemplo, en el campo de la terapia cognitiva (Segal, Teasdale y Williams, 2002), y en terapia de conducta (Hayes, 2004), como una forma diferente de enfocar y tratar los problemas de los seres humanos, se ha difundido por todo el mundo. En la segunda década del Siglo XXI, la práctica del mindfulness se utiliza en muchas áreas, desde hospitales a institutos de terapia, en los negocios, en las grandes compañías en Internet, etc. (Brandsma, 2017). Existe una gran variedad de posibilidades "mindful...". Las técnicas de la conciencia plena nos rodean, no sólo en contextos académicos, sino también en los medios de comunicación. Trabajar en mindfulness está de moda. Una nueva sociedad que divulga estas técnicas de conciencia plena, y éstas contribuyendo de una forma distintiva a la sociedad. Por ejemplo, con una comprensión diferente de las guías éticas para los terapeutas.

En este sentido, existen grandes diferencias entre lo que se puede considerar una guía ética en una perspectiva Occidental vs. Oriental. Según Morgan (2013) un terapeuta debe seguir determinados preceptos, más relacionados con la acción moral que con la ética, tal y como se ha definido previamente. Así, un terapeuta debería seguir normas como no matar (comportarse con compasión y reverenciar la vida); no robar (estar más preocupado por la corrección y la generosidad); no llevar a cabo una conducta sexual inadecuada (manejar la energía sexual con habilidad); no mentir (emplear un habla sincera y habilidosa); y, finalmente, no tomar drogas/fármacos, etc. (ser conscientes sobre el consumo).

Tenemos un segundo ejemplo, si se asume que la práctica de la conciencia plena, basada sobre la psicología Budista, no diferencia entre acciones "buenas" y "malas" ya que éstas son, simplemente, convenciones sociales. Si detener el sufrimiento es la meta general de la práctica del mindfulness (Segovia, 2017), entonces, y según Germer (2013), se debe diferenciar entre acciones "beneficiosas" en todos los sentidos (aquellas que disminuyen el sufrimiento) y las acciones "no beneficiosas" (aquellas que incrementan nuestro sufrimiento). Sin ninguna duda, esto supone una perspectiva distinta sobre cualquier meta psicoterapéutica, con requisitos éticos diferentes.

Cualquier explicación sociocultural debe tener en cuenta no sólo los elementos sociales de cada momento histórico, sino la reflexión que se debe hacer sobre el tipo de sujeto que se desarrolla y surge en dicha sociedad. Por tanto, cualquier persona que aplica o recibe terapia, posee unas características específicas. A la base de cada modelo existe una imagen única del ser humano (Pilgrim, 1997; Rychlak, 2003), al igual que existe en cualquier taxonomía, por ejemplo, el DSM (Cushman, 2002).

Así, y siguiendo con nuestro ejemplo anterior, el ser humano detrás de la práctica de la conciencia plena es muy diferente de la imagen que se tendría de éste en la práctica psicoterapéutica Occidental. Un terapeuta que emplee el mindfulness 
debe entender que está asumiendo que existe la posibilidad de dirigir su trabajo a un tipo de sujeto distinto. Esto es, aquel que puede no ejercer control (por ejemplo, en la práctica del mindfulness en la terapia de aceptación y compromiso; Hayes, 2004), vs. uno que sí que puede y debe hacerlo (como en el trabajo en terapias cognitivas clásicas).

Sin embargo, sería conveniente asumir que el surgimiento de esta tendencia, cómo se mantiene, y qué defiende como apropiado para un cliente en concreto, carece, en buena medida, de una reflexión específica sobre los valores, perspectivas, tipo de sujeto implícito, etc., a su base. Indudablemente, se debe reconocer que esta es una dicotomía muy simplificada que no puede integrar las variadas y numerosas características de la práctica Occidental $v s$. Oriental. La empleamos en este contexto sólo para ayudarnos en nuestra explicación y, simplemente, como un breve ejemplo de lo difícil que es evitar la ética (basada sobre nuestra definición del inicio) en el campo psicoterapéutico y de la dependencia sociocultural de las guías éticas terapéuticas.

En definitiva, podemos asumir que los enfoques psicoterapéuticos integran supuestos explícitos e implícitos, construidos socialmente, sobre cuáles son las metas y los métodos de la psicoterapia y aquello que es correcto hacer. Esto nos ofrece el contexto para la negociación de los roles terapéuticos y cómo se resuelve, de forma diferente, el poder inherente, a través de la relación terapéutica. Igualmente, nos facilita conocer cuál debe ser el foco psicoterapéutico, y cómo se organiza el trabajo conjunto cliente-terapeuta. Todo ello incide totalmente en el campo de la ética y, por tanto, es importante que forme parte de la formación de los terapeutas.

\section{Los tres desafíos éticos de la psicoterapia}

Pope y Vasquez (2011) destacaron que los mayores desafíos éticos para los psicólogos se relacionaban con la confianza, el poder y el ofrecer cuidados, estando los tres interconectados. Por ejemplo, la confianza es básica y facilita un contexto a los clientes desde el cual explorar sus quejas, justificando y aceptando la intrusión del terapeuta en sus problemas. Al ser una profesión regulada, la terapia depende de la sociedad, y la sociedad depende de los terapeutas para que se hagan cargo de los problemas de los clientes. "A cambio de asumir un papel en el que la seguridad, el bienestar y el beneficio último de los clientes se consideran una confianza sagrada, el terapeuta tiene el derecho de asumir los roles, privilegios y el poder debidos" (Pope y Vasquez, 2011, p. 34).

Por tanto, los terapeutas obtienen su poder por la confianza que los clientes y la sociedad les otorgan, lo que correlaciona con el cuidado de los terapeutas hacia sus clientes y su única meta: aumentar el bienestar de los clientes.

Es importante señalar que existe un poder diferencial en terapia. Aunque muchos enfoques psicoterapéuticos destacan la necesidad de seguir una perspectiva de iguales, observada de maneras diferentes en la relación terapéutica, la cuestión del poder (sobre todo, como una preocupación ética) va más allá de esta relación 
(Pope y Vasquez, 2011). Por ejemplo, la relación terapéutica en la terapia centrada en el cliente (Rogers, 1957) se puede diseñar desde una perspectiva de igualdad. Sin embargo, el poder, o las diferencias de poder estarían todavía presentes en la terapia. Un participante es el terapeuta, y otro es el cliente, que paga o no (dependiendo del contexto) por el tratamiento profesional que recibe. Entonces, "un atributo definitorio del profesional es el reconocimiento, comprensión y manejo cuidadoso del considerable poder-y de la responsabilidad personal por dicho poder-inherentes a su papel" (Pope y Vasquez, 2011, p. 39).

\section{La confianza en psicoterapia}

¿Por qué los terapeutas están legitimados para ejercer dicho papel con sus privilegios como profesionales en salud mental? ¿Por qué los clientes confían en ellos para resolver sus problemas? En primer lugar, porque la psicoterapia es una profesión regulada (véase, por ejemplo, una revisión de las normativas europeas, Van Broeck y Lietaer, 2008). Desde sus inicios, la psicoterapia está socialmente sancionada porque las universidades, instituciones, sociedades profesionales, revistas, etc. han dedicado sus esfuerzos a establecer las reglas de una práctica correcta. Por ejemplo, desde el interés de Freud por evitar el "psicoanálisis salvaje" o las regulaciones del Modelo de Boulder, ha existido un interés continuo en la formación y en establecer quién y por qué medios puede ser terapeuta. Esto es básico para la práctica ética, ya que la formación no puede evitar responder a lo que es correcto hacer, o no hacer, en este campo.

La importancia de la formación se observa en todos los enfoques psicoterapéuticos. Por ejemplo, la psicoterapia cognitiva se ha preocupado mucho por describir cuáles son las características deseables de un terapeuta cognitivo (e.g.: Newman, 2010; Rodolfa et al., 2005), y cuál es la mejor manera de emplear esta terapia. Estos trabajos regulan cómo hacer terapia cognitiva, influyendo, sin ninguna duda, en la confianza de los clientes en sus psicoterapeutas cognitivos. En el contexto cognitivo, una buena formación ha sido importante desde sus inicios y cada vez es más relevante (Bennett-Levy, 2006).

En segundo lugar, el supuesto sobre la forma correcta de ser un terapeuta profesional es una herencia de la Modernidad (McNamee y Shawver, 2004). Gracias a características modernistas como la industrialización, la vida en las ciudades y la secularización, apareció un interés nuevo, según Cushman (1992), en el mundo físico, las humanidades, la ciencia, el comercio y la racionalidad. Por tanto, el Estado desarrolló formas de controlar a un nuevo tipo de sujeto, siempre cambiando y desarrollándose, menos regulado y limitado por el papel de la religión y, por ello, menos predecible. Este nuevo sí mismo, más aislado, menos comunitario y más individualista, y más confundido sobre lo que estaba bien o mal, sobre lo que era ético o no, necesitaba de la aparición de un nuevo tipo de experto, el filósofo moderno que, con el tiempo, se convirtió en un científico social el cual desarrolló técnicas para observar, medir, predecir y controlar la conducta de los sujetos 
(Foucault, 1975).

Los terapeutas se corresponden con esta figura, creada socialmente, y convertida en una parte integral de la tradición modernista. Tal y como planteó McLeod (1997), "la psicoterapia es una forma cultural o el lugar en el que la gente tiene permitido contar sus historias y problemas personales, delante de, o ayudados por otra persona con habilidades y estatus especiales para esta tarea" (p. 20).

Siguiendo a Berger y Luckmann (1966):

"La terapia supone la aplicación de la maquinaria conceptual para asegurarnos de que los que se desvían, real o potencialmente, permanecen dentro de las definiciones institucionalizadas de la realidad, o por decirlo de otra manera, evita que los 'habitantes' de un universo determinado 'emigren' de él. Lo hace aplicando el dispositivo legitimado para los 'casos' individuales. Puesto que, como hemos visto, cada sociedad tiene que hacer frente al peligro de la desviación del individuo, podemos asumir que la terapia, de una manera u otra, es un fenómeno social, global. Sus acuerdos institucionales especificos, desde el exorcismo al psicoanálisis, desde el consejo religioso a los programas de consejo personal, pertenecen, sin ninguna duda, a la categoría del control social" (p. 130).

Si traspasamos este punto de vista a la psicoterapia, el marco modernista ha contribuido a construir un bagaje de conocimiento, difundido a nivel mundial, que ofrece a la sociedad y a los posibles usuarios de la psicoterapia, guías para saber qué deben pedir y dónde y cómo encontrar un terapeuta. La terapia se ha globalizado. Millones de personas en las naciones y sociedades Occidentales industrializadas poseen cierto conocimiento sobre lo que un psicólogo o un terapeuta pueden hacer por ellas. Estas nociones, más o menos específicas, se adquieren en la escuela, la TV, las revistas, las películas, etc. (McLeod, 1997). Estas guías y conocimiento sobre la terapia, socialmente construidas, facilitan que los clientes confíen en sus terapeutas ya que se dispone de un marco social que le da al terapeuta el poder para curar al estar su profesión socialmente regulada. Es decir, la sociedad depende de los terapeutas para "cumplir con la confianza dirigida hacia el beneficio de los clientes al igual que al orden social" (Pope y Vasquez, 2011, p. 34).

Como destacó Howe (1993) 'existe un 'centro', un sujeto individual que posee un acceso privilegiado a la verdad y que guarda la llave única que puede abrir los contenidos de la experiencia del cliente" (p. 185). Así, un futuro terapeuta debe saber que ser el guardián de esta llave conlleva una gran responsabilidad ética.

El rol del terapeuta está sancionado socialmente, al igual que el de los clientes. Es decir, puede ser una cuestión ética, pero en una vía de dos sentidos. Por ejemplo, tanto clientes como terapeutas tienen expectativas terapéuticas sobre el cambio y sobre qué obtener de ese proceso.

Por ejemplo, los clientes pueden esperar que sus terapeutas cumplan con sus expectativas. Esto es importante ya que se puede relacionar con el resultado en terapia (Bohart y Greaves Wade, 2013). Igualmente, los psicoterapeutas pueden 
tener expectativas relacionadas con el resultado (Connor y Callahan, 2015). Siguiendo a Feltham (2017):

“... la atención recibida de un sanador, socialmente sancionado, y en un ámbito regulado, tiene una gran influencia en el establecimiento de expectativas positivas. A la gente le tranquiliza estar en el ambiente adecuado, tratados profesionalmente, con las cualificaciones adecuadas, y con un fundamento explícito para la terapia" (pp. 151-152).

Es decir, que cualquier terapia implica ofrecer a los clientes una sensación de esperanza, de recibir ayuda (Frank, 1974). Indudablemente, esto no significa que todos los clientes (ni los terapeutas) acuden a psicoterapia con el mismo nivel de expectativas. Algunos clientes pueden tener una fe positiva en el resultado, otros pueden estar ambivalentes, y algunos desconfiar del resultado terapéutico (Glass, Arknoff y Shapiro, 2001).

Aunque muchos posibles clientes pueden pensar que no necesitan un terapeuta, llegándose, incluso, a describir o anticipar prácticas no psicoterapéuticas (Gergen, 2000; Parker, Georgaca, Harper, McLaughin y Stowell-Smith, 1995), la mayoría de ellos tiene cierta información para elegir uno u otro tipo de enfoque terapéutico. Si se instruye a los clientes sobre lo que esperar de la terapia y sobre aquello que mantiene todo el dispositivo terapéutico, probablemente, harán elecciones mejor informadas y se ajustarán mejor con sus terapeutas (Glass, Arknoff y Shapiro, 2001). Se ha visto que los clientes acuden a psicoterapia con un conocimiento, obtenido de Internet y los libros de auto-ayuda, que puede llevar a que traten a los psicoterapeutas como simples asesores y no como figuras de autoridad, como eran en el siglo pasado (Strong y Sutherland, 2007). Por tanto, la evolución de la sociedad supone una exigencia y una actitud diferente hacia estos "científicos sociales".

Como planteó McLeod (2012):

“... los miembros de sociedades Occidentales están expuestos a un rango de discursos y prácticas sobre cómo dar sentido y resolver las dificultades emocionales, comportamentales, y relacionales. Asi, probablemente, cuando una persona acude a terapia estará influida por algunos discursos y prácticas, en relación a su sensación general sobre aquello que le puede ser útil terapéuticamente. Además, tendrá determinadas preferencias sobre el valor de ciertas ideas y métodos terapéuticos, mientras que considerará otros enfoques como carentes de credibilidad" (pp. 23-24).

Sin embargo, los terapeutas son capaces de tomar sus decisiones terapéuticas sobre aquello que piden a sus clientes porque el rol de éstos está sancionado socialmente. Los clientes confían en sus terapeutas y acuden a terapia pudiendo hablar sobre cosas que no le dirían a nadie más, permitiendo que sus terapeutas les hagan todo tipo de preguntas intrusivas (Pope y Vasquez, 2011). ¿Por qué hacen esto los terapeutas? ¿Por qué es correcto inmiscuirse en las áreas más profundas y personales de los clientes? 
En primer lugar, porque la psicoterapia ha heredado la idea modernista sobre la necesidad de un científico social, con un papel clave. La metáfora del Panóptico, planteada por Foucault (1975) sería el símbolo perfecto (según Cushman, 1992) de este nuevo orden, necesario desde la perspectiva modernista, tal y como se ha descrito anteriormente. El Panóptico era una prisión, diseñada por Bentham en el siglo XVIII. Su característica principal era que el prisionero podía verse a sí mismo, pero nunca a sus carceleros. La metáfora del Panóptico describe cómo los prisioneros, a) aumentaban su tolerancia para ser observados; b) su tendencia y habilidad para observarse a ellos mismos; c) la presión por ser normales; $y, d$ ) aumentaban sus intentos para practicar la auto-observación y el cambio comportamental consciente.

Esta metáfora nos ofrece una descripción clara sobre el tipo de sujeto que demanda este nuevo científico social. Este tipo de sujeto puede justificar, como apropiado, que el terapeuta se inmiscuya en su área más privada. Para hacer posible la práctica de la psicoterapia necesitamos un individuo que sea capaz de ser autoconsciente y con capacidad introspectiva (Caro Gabalda, 2015). Por tanto, cualquier práctica correcta debe facilitar el acceso al mundo privado, a un tipo de sujeto que espera que lo observen (Cushman, 1992). Los individuos pueden observarse a ellos mismos, preguntar sobre lo que son, pensar sobre lo que esconden o revelan, y especular sobre sus características e identidad verdaderas.

El terapeuta dirige su trabajo hacia este tipo de sujeto, al margen del enfoque que emplee. Aunque podemos establecer diferencias entre modelos, en cualquier caso se necesita un terapeuta que confíe en las posibilidades del cliente para la autoconciencia, la auto-exploración, etc. Por ello, es fundamental que el terapeuta sea capaz de entrar en el mundo del cliente. Los clientes confían en sus terapeutas para comportase de una manera concordante con ello.

\section{El cuidado en psicoterapia}

Es una responsabilidad ética emplear el poder y la confianza que se les da a los terapeutas en relación a una única meta, el bienestar del cliente. Prestar cuidados es la base de las responsabilidades del terapeuta (Pope y Vasquez, 2011). De esta manera, los terapeutas sustentan su papel en función del beneficio de los otros. Por dicho motivo, esta meta se incluye en los Códigos Deontológicos. Beneficiar a otras personas es una demanda lógica $\mathrm{y}$, por tanto, un principio ético importante (Tjeltveit, 2006).

Según Howe (1993) los clientes cuentan una historia sobre sus experiencias terapéuticas caracterizada por tres temas principales: acéptame, compréndeme y habla conmigo. Además, los clientes describen al terapeuta "agradable" en función de seis temas: aquel que conforta; los buenos terapeutas son gente real; la calidad de la relación; gustar y gustarles; verdad y honestidad; y apoyo y estar allí (Howe, 1993, p. 21). Por tanto, los futuros psicoterapeutas deben darse cuenta de que deben satisfacer estas expectativas de los clientes. Por otro lado, esto no es una tarea fácil. La práctica del terapeuta debe guiarse por pensar y planificar cuidadosamente qué 
hacer en psicoterapia. Su responsabilidad no recae, solamente, en estar bien formados, como una justificación para la confianza y el cuidado. Cuidar del cliente no se puede desarrollar sin una sintonización sensible y responsiva (Stiles, HonosWebb y Surko, 1998) a las demandas del cliente. Este trabajo no se puede llevar a cabo sin dolor ni sufrimiento. Por decirlo de otra manera, los clientes primero se sienten peor, para después sentirse mejor (Stiles, Osatuke, Glick y McKay, 2004). Por consiguiente, los terapeutas deben cuidar del cliente considerando, cuidadosamente, sus consecuencias.

De esta manera, podemos asumir que un buen terapeuta es aquel capaz de profundizar en las necesidades de su cliente y tratarlas adecuadamente. Sin embargo, esto significa que los clientes deben sentirse cómodos en terapia, confiando en sus terapeutas, como una forma de justificar profundizar en su sufrimiento (o, sentirse peor, antes de sentirse mejor). Así se abre la puerta a la complejidad de sus vidas (Messer, 2006). La exigencia ética de dar cuidados se logra, sólo, cuando esta puerta se abre.

Hay que cuidar cómo tratamos las necesidades de los clientes, ya que las personas que acuden a terapia están en un estado de vulnerabilidad, o como Frank (1974) afirmó, desmoralizados. Esta vulnerabilidad no sólo se relaciona con describir al cliente como "roto", como un "rompecabezas", como "vacío", etc, o como un "alma fragmentada" que necesita ayuda y una guía cuidadosa (Caro Gabalda, 2018). Tiene que ver también con elementos tan importantes para el contexto de la ética, que requieren tratarse con cuidado. Los clientes son la parte que confía, que presenta el problema, que revela información confidencial, idealiza a sus terapeutas, y sufre estrés por el proceso de tratamiento (Gutheil, Jorgenson y Sutherland, 1992).

Aunque se permite que los terapeutas profundicen en el alma del cliente, su bienestar se define de manera diferente según el enfoque. Esto significa que cada foco terapéutico, en diferentes modelos, se construye de forma distinta.

Como ejemplo, podemos comparar las diferencias sobre cómo se resuelve el tema de la razón y la emoción en el campo de las psicoterapias cognitivas. Las terapias modernistas tradicionales, como las de reestructuración, tienden a favorecer la razón frente a la emoción, mientras que las constructivistas favorecen la emoción frente a la razón (Caro Gabalda, 1995b; Mahoney, 1991). Es decir, hay un conflicto entre la tradición filosófica del Romanticismo en la que las emociones son el foco de respeto y preocupación, con la tradición de la Ilustración que considera que las emociones deben regularse (Fowers, 2005). Igualmente, esto se puede relacionar con la manera de tratar emociones y pensamientos en las terapias clásicas (¡logremos su control!) vs. cómo se tratan, como se ha comentado anteriormente, en el mindfulness y en los enfoques contextuales, o en el constructivismo, donde se conceptualizan las emociones como importantes fuentes de conocimiento (Mahoney, 1991). De nuevo, un ejemplo de un foco diferente, apropiado o no según el enfoque.

Estas diferencias sobre razón vs. emoción se pueden comprender desde una 
perspectiva ética que implica, al mismo tiempo, un punto de vista sobre un tipo de persona diferente. Si seguimos a Richardson (2012) en sus reflexiones sobre la ética de la virtud, establecida por Fowers (2005), podemos trascender esta dicotomía. Dice Richardson (2012):

"Se supone que las emociones revelan el "tipo de persona que uno es" (Fowers, 2005, p. 44) y el estado actual de sus "puntos fuertes de carácter" (p. 9) o las excelencias vitales. El desarrollo del carácter implica educar las propias emociones, sin juzgar y sin coaccionar, de manera "que sean consistentes con actuar bien" y con organizar una parte importante de la experiencia sobre lo que uno considera que es el mejor tipo de vida. Aquella persona que haya cultivado tales excelencias caracterológicas puede actuar "con la emoción, el pensamiento y la acción estando en concordancia", haciendo posible un grado de "continuidad, totalidad, y crecimiento" (p. 66) a lo largo de su vida" (p. 28).

Finalmente, no podemos olvidar que la terapia es una teoría. Brevemente, esto significa que está relacionada con elementos culturales y sociales importantes sobre los seres humanos. Como una teoría sobre los seres humanos (Robinson, 1997; Rychlak, 2003) la terapia reflexiona sobre el tipo de vida que los seres humanos deben vivir; cuál es el "mejor" tipo de vida para ellos; cuál es el tipo de identidad que deben buscar y promulgar; los medios permitidos para que algunas personas influyan en las vidas de los demás, y cuáles pueden ser, finalmente, las metas terapéuticas adecuadas. Sin embargo, esta cuestión sobre lo que es bueno o malo se responde de maneras diversas, aunque, desde nuestra perspectiva, está basada sobre un importante supuesto. De esta manera, la terapia es más que el tratamiento de los síntomas de los clientes o de sus trastornos (González Pardo y Pérez Álvarez, 2007; Smith, 2009). Entonces, las cuestiones teóricas y filosóficas implícitas se deben aclarar, en lugar de darlas por sentado (Pérez Álvarez y García Montes, 2017; Woolfolk y Richardson, 2008). Lo que es bueno, malo, cuáles son las obligaciones, virtudes, etc. de los terapeutas, relacionado todo ello con el cuidado de sus clientes, son elementos centrales de la práctica psicoterapéutica (Tjeltveit, 2004).

\section{El poder en psicoterapia}

Tal y como se ha definido en los apartados anteriores, a los terapeutas se los trata como estando capacitados para su papel y como profesionales cuyas sugerencias, directrices o guías se deben seguir. Los terapeutas poseen las licenciaturas adecuadas, una formación específica y una licencia profesional que aumenta su credibilidad y autoridad, facilitándoles la posibilidad de servir como agentes del cambio comportamental (Foote, 2011).

Los terapeutas deben usar su poder con destreza al cuidar de sus clientes y al ser responsables de la confianza que los clientes y la sociedad les otorgan (Pope y Vasquez, 2011). Esto es muy relevante cuando “..... al terapeuta se lo nombra y se lo percibe como el que está cualificado, el que sabe, el que posee una super-salud, 
cuyos poderes, aparentemente, bordean la telepatía, y que tiene la llave para salir de la desgracia" (Feltham, 2017, p. 150).

Por tanto, los clientes pueden considerar que sus terapeutas poseen un gran poder. Este poder se observa en áreas diferentes. El poder para adscribir palabras y definir, o el poder para adscribir etiquetas diagnósticas, es especialmente relevante. Como describió Cushman (1992):

"cada sociedad o cada era se puede estudiar según los juicios históricos relacionados con, a) la configuración predominante del sí mismo, b) las enfermedades que afectan, de forma característica, al si mismo, c) las instituciones o medios oficiales responsables de la cura de dichas enfermedades, $y$ d) las tecnologías que las instituciones privadas o los que practican la psicoterapia han empleado para curar las enfermedades características de este sí mismo" (p. 24).

Todo ello se deriva de que la causa o la explicación de los problemas de los seres humanos, cómo evaluar y cómo superar estos problemas está a la base de las teorías y de las decisiones terapéuticas (Gergen y Kaye, 1992). Así, el poder para adscribir etiquetas diagnósticas debe emplearse a partir de una reflexión ética, profunda que, desde el punto de vista reflejado en este artículo, se puede desarrollar mejor si situamos la comprensión de las decisiones psicoterapéuticas en un contexto social y cultural.

Por ejemplo, en función de la clase social, la cleptomanía se consideraba un trastorno mental cuando la mujer pertenecía a una clase social alta, y un robo cuando la mujer pertenecía a una clase baja. La construcción de la misma situación era diferente según la clase social (Parker et al., 1995). La historia de la psicopatología y de la psicoterapia está plagada de tales ejemplos (véase, Woolfolk y Murphy, 2004; Woolfolk y Richardson, 2008).

Higginbotham, West y Forsyth (1988) plantearon que cualquier enfermedad o cualquier serie de síntomas psicológicos eran parte de la experiencia humana y se convertían en objetos de la acción humana, sólo cuando las personas les adscribían significado, a través del lenguaje. En segundo lugar, cada categoría de enfermedad es un producto cultural, basada sobre las teorías y las redes de significado empleadas para interpretar y comunicar los síntomas específicos. Finalmente, cada práctica clínica se sustenta sobre una interpretación. Cada terapeuta, con independencia de su enfoque, comprende y enmarca los síntomas, experiencias, conductas, etc., de los clientes. El terapeuta sitúa el foco en algunos elementos. Algunos se consideran básicos o centrales, otros marginales, y otros como no tan relevantes. Se hacen abstracciones y el terapeuta se implica en un proceso continuo de traducción a través de distintos sistemas de significado. Como muestra la historia de la psicoterapia (Robinson, 1976), esto depende del momento histórico concreto. Esta cuestión afecta al terapeuta y al cliente, por igual. Los clientes interpretan los significados que los terapeutas dan a sus problemas, lo que les facilita construir nuevos significados sobre lo que les está sucediendo. 
Por tanto, cada práctica psicoterapéutica no puede separarse de un contexto de significados negociados, tal y como muestran los códigos éticos. Por ejemplo, desde la perspectiva de la ética conversacional (Strong y Sutherland, 2007) las conversaciones y diálogos de los clientes y los terapeutas, y el trabajo terapéutico conjunto deben sintonizarse adecuadamente. Es decir, la práctica ética supone no sólo usar nuestro conocimiento profesional, sino llevarla a cabo, tal y como Stiles et al. (1988) definieron, de manera responsiva. Además, esto no puede separarse del lenguaje. Es decir, la práctica psicoterapéutica se asocia con una ética del contenido (lo que se debe, o no, hacer; por ejemplo, romper la confidencialidad), y también con una ética conversacional (un razonamiento práctico y formas de hablar con los clientes específicas a cada situación). Entonces,

“.... Los psicólogos trabajan de forma relacional y contextualmente responsivas, sustentadas en su ética y su conocimiento.... Los psicólogos, en su interacción profesional, se enfrentan a tensiones éticas al balancear su conocimiento profesional e intenciones, con las intenciones y preferencias de los clientes" (Strong y Sutherland, 2007, p. 95).

En consecuencia, los terapeutas deben reconocer que su poder implica nombrar, definir y seleccionar las mejores maneras de trabajar con los problemas de los clientes. Por tanto, los terapeutas tienen el poder del conocimiento y de qué factores afectan a los seres humanos y la mejor manera de producir cambios en ellos (Pope y Vasquez, 2011).

\section{Conclusión}

Según Owen (1992) los terapeutas se relacionan con sus clientes desde sus explicaciones terapéuticas, que ofrecen un contexto, implícito y que se da por sentado o justificado, en relación a su práctica. Las teorías psicológicas y psicoterapéuticas, al igual que cualquier otro tipo de teoría, no son perfectas. Si no se reconocen los problemas dentro de ellas, permanecerán, sin duda, incuestionados.

Además, el poder socialmente adscrito a los terapeutas facilita que éstos estén en terapia como la persona fuerte, frente al cliente que sería la débil (Avia Aranda, 2018; Gergen y Kaye, 1992). Este supuesto puede sesgar el trabajo terapéutico y tiene una gran influencia sobre cómo manejan los terapeutas su poder y cómo justifican su práctica con la única meta de ofrecer un contexto de confianza y de búsqueda del bienestar y cuidado, profundos, de los clientes.

Muchas teorías psicoterapéuticas pueden fracasar si no se reflexiona críticamente sobre las acciones de los terapeutas en las vidas de sus clientes. Además, muchos terapeutas pueden fallar si no hacen tal tipo de reflexión. Curiosamente, los terapeutas no suelen reflexionar sobre sus teorías y sobre cómo se construyen, cuando, con mucha frecuencia, piden a sus clientes que cambien las suyas.

La respuesta a la pregunta de por qué el terapeuta puede situarse desde una perspectiva de autoridad es porque el conocimiento es un producto social. Es decir, 
como la historia y la evolución de lo que es la salud mental y la forma de enfocar dichos problemas nos enseña y recuerda, las representaciones científicas son una consecuencia de la comunidad científica que negocia, compite o conspira y acuerda. No debemos tomar como inequívocos nuestros mapas terapéuticos.

La formación adecuada de los terapeutas es una de las principales responsabilidades éticas de los implicados en ella. Brevemente, esta formación debe incluir la parte técnica, así como la teórica, o las teorías sobre su trabajo y sobre cómo aplicar dicho conocimiento. Sin embargo, debe existir un nivel más elevado de abstracción y de reflexión sobre el contenido y el contexto de esta formación. Los terapeutas deben reflexionar sobre sus antecedentes, o sobre los orígenes históricos y socioculturales de su trabajo, que lo justifican y lo facilitan con sus consecuencias implícitas y explícitas. Hay una necesidad ética de pensadores más críticos.

\section{Referencias bibliográficas}

Avia Aranda, Ma D. (2018). "La casa de juegos” o la importancia en la terapia de la vida personal del terapeuta. En I. Caro Gabalda (Ed.), Cine, metáforas y psicoterapia (pp. 97-106). Madrid, España: Pirámide.

Bennett-Levy, J. (2006). Therapist skills: A cognitive model of their acquisition and refinement. Behavioural and Cognitive Psychotherapy, 34, 57-78. https://doi.org/10.1017/S1352465805002420

Berger, P. L. y Luckmann, T. (1966). The social construction of reality. Londres, Reino Unido: Penguin.

Bohart, A. C. y Greaves Wade, A. (2013). The client in psychotherapy. En M. J. Lambert (Ed.), Bergin and Garfield's handbook of psychotherapy and behavior change (6 $6^{\mathrm{a}} \mathrm{ed}$.) (pp. 219-257). Nueva Jersey, NJ: John Wiley and Sons.

Brandsma, R. (2017). The mindfulness teaching guide. Oakland, CA: New Harbinger Publications.

Caro Gabalda, I. (1995a). La psicoterapia en una sociedad postmoderna. Revista de Psicoterapia, 6(24), 29-44. Recuperado de: https://bit.ly/2JyBp2c

Caro Gabalda, I. (1995b). Pasado, presente y futuro de las psicoterapias cognitivas. Boletín de Psicología, 46, $115-$ 160.

Caro Gabalda, I. (1999). Un enfoque postmoderno sobre la salud mental y la psicoterapia. Revista de Psicoterapia, 10(37), 31-50. Recuperado de: https://bit.ly/2LdkLsa

Caro Gabalda, I. (2015). The subject in cognitive psychotherapy. Anales de Psicología, 31, 379-389. https:// doi.org/10.6018/analesps.31.2.200201

Caro Gabalda, I. (2018). Conclusión: Cine, metáforas y psicoterapia. En I. Caro Gabalda (Ed.), Cine, metáforas y psicoterapia (pp. 317-338). Madrid, España: Pirámide.

Clarkson, P. (2005). Values, ethics and the law: a story with some morals. En N. Tottom (Ed.), The politics of psychotherapy (pp. 97-107). Maidenhead, Reino Unido: Open University Press.

Connor, D. R. y Callahan, J. L. (2015). Impact of psychotherapist expectations on client outcomes. Psychotherapy, 52, 351-362. https://doi.org/10.1037/a0038890

Consejo General de Colegios Oficiales de Psicólogos (COP, 2018). Código Deontológico del Psicólogo. Recuperado de: http://www.cop.es/index.php?page=codigodeontologico

Consumer Reports (1995, Noviembre). Mental health: Does therapy help? 734-739.

Cushman, P. (1992). Psychotherapy to 1992: A historically situated interpretation. En D. K. Fredheim (Ed.), History of psychotherapy (pp. 21-64). Washington, DC: APA.

Cushman, P. (2002). How psychology erodes personhood. Journal of Theoretical and Philosophical Psychology, 22, 103-113. http://dx.doi.org/10.1037/h0091217

David, D., Cristea, I. y Hofman, S.G. (2018). Why cognitive behavioral therapy is the current gold standard of psychotherapy. Frontiers in Psychiatry, 9, 1-3. https://doi.org/10.3389/fpsyt.2018.00004

Elliott, R.(1998). Editor's introduction: A guide to the empirically supported treatments controversy. Psychotherapy Research, 8(2), 115-125. https://doi.org/10.1080/10503309812331332257 
Elliott, R. (2008). A linguistic phenomenology of ways of knowing and its implications for psychotherapy research and psychotherapy integration. Journal of Psychotherapy Integration, 18, 40-65. https:// dx.doi.org/10.1037/1053-0479.18.1.40

Feltham, C. (2017). Client experiences. En C. Feltham, T. Hanky y L. A. Winter (Eds.), The Sage handbook of counselling and psychotherapy (pp. 149-159). Londres, Reino Unido: Sage.

Foote, W.E. (2011). On duty, power and informed consent in relation to termination in psychotherapy. Professional Psychology, 42, 165-167.

Foucault, M. (1975). Surveiller and punir. Paris, Francia: Gallimard.

Fowers, B. (2005). Virtue ethics and psychology: Pursuing excellence in ordinary practice. Washington, DC: APA.

Frank, J. D. (1974). Psychotherapy: The restoration of morale. American Journal of Psychiatry, 131, 271-274. https://doi.org/10.1176/ajp.131.3.271

Frank, J. D. y Frank, J.B. (1991). Persuasion and healing ( $3^{\mathrm{a}}$ Ed). Baltimore, MD: The Johns Hopkins University Press.

Gabriel, L. (2005). Speaking the unspeakable. The ethics of dual relationships in counselling and psychotherapy. Londres, Reino Unido: Routledge.

Gergen, K. G. (1991). The saturated self. Nueva York, NY: Basic Books.

Gergen, K. G. (2000). The coming of creative confluence in therapeutic practice. Psychotherapy, 37, 364-369. https://dx.doi.org/10.1037/0033-3204.37.4.364

Gergen, K. G. y Kaye, J. (1992). Beyond narrative in the negotiation of meaning. En S. McNamee y K. G. Gergen (Eds.), Therapy as a social construction (pp. 166-187). Londres, Reino Unido: Sage.

Germer, C. K. (2013). Mindfulness. What is it? What does it matter? En C. K. Germer, R. D. Siegel y P. R. Fulton (Eds.). Mindfulness and psychotherapy (2" ed.) (pp. 3-35). Nueva York, NY: Guilford Press.

Glass, C. R., Arnkoff, D. B. y Shapiro, S. J. (2001). Expectations and preferences. Psychotherapy, 38, $455-461$. http://dx.doi.org/10.1037/0033-3204.38.4.455

González Pardo, H. y Pérez Álvarez, M. (2007). La invención de trastornos mentales. Madrid, España: Alianza Editorial.

Gutheil, T., Jorgenson, L. y Sutherland, P. (1992). Prohibiting lawyer-client sex. Bulletin of the Academy of Psychiatry and Law, 20, 365-382.

Hayes, S. C. (2004). Acceptance and commitment therapy, relational frame theory, and the third wave of behavioural and cognitive therapies. Behavior Therapy, 35, 639-665. https://bit.ly/2XIhUNN

Henry, W. P. (1998). Science, politics, and the politics of science: The use and misuse of empirically validated treatment research. Psychotherapy Research, 8, 126-140. https://doi.org/10.1080/10503309812331332267

Higginbotham, H. N., West, S. G. y Forsyth, D. R. (1988). Psychotherapy and behavior change. Social, cultural and methodological perspectives. Nueva York, NY: Pergamon Press.

Howe, D. (1993). On being a client. Londres, Reino Unido: Sage.

Jenkins, P. (2002). Legal issues in counselling and psychotherapy. Londres, Reino Unido: Sage.

Kabat-Zinn, J. (1990). Full catastrophe living: Using the wisdom of your body and mind to face stress, pain, and illness. Nueva York, NY: Delta.

Kabat-Zinn, J. (1999). Indra's net at work: The mainstreaming of dhamma practice in society. En G. Watson, S. Batchelor y G. Claxton (Eds.), The psychology of awakening: Buddhism, science and our day-to-day lives (pp. 225-249). Londres, Reino Unido: Rider.

Koocher, G. P. (2007). Twenty-first century ethical challenges for psychology. American Psychologist, 62, $375-$ 384. http://dx.doi.org/10.1037/0003-066X.62.5.375

Kvale, S. (1992). Postmodern psychology: A contradiction in terms? En S. Kvale (Ed.), Psychology and postmodernism (pp. 31-57). Londres, Reino Unido: Sage.

Mahoney, M. J. (1991). Human change processes. Nueva York, NY: Basic Books.

McLeod, J. (1997). Narrative and psychotherapy. Londres, Reino Unido: Sage.

McLeod, J. (2001). Qualitative research in counselling and psychotherapy. Londres, Reino Unido: Sage.

McLeod, J. (2012). What do clients want from therapy? A practice-friendly review of research into clients preferences. European Journal of Psychotherapy and Counselling, 14, 19-32. https://bit.ly/2XO3qMj

McNamee, S. y Shawver, L. (2004). Therapy as social construction. Back to basics and forward toward changing issues. Sheila McNamee with conversational partner Lois Shawver. En T. Strong y D. Paré, Furthering talk (pp. 253-270). Nueva York, NY: Springer.

Messer, S. B. (2006). What qualifies as evidence of effective practice?: Patient values and preferences. En J. C. Norcross, L. E. Beutler y R. F. Levant (Eds.), Evidence-based practices in mental health (pp. 32-39). Washington, DC: APA. 
Morgan, S. P. (2013). Practical ethics. En C. K. Germer, R. D. Siegel y P. R. Fulton (2013). Mindfulness and psychotherapy (pp. 112-129). Nueva York, NY: Guilford Press.

Newman, C. F. (2010). Competency in conducting cognitive-behavioral therapy: Foundational, functional, and supervisory aspects. Psychotherapy, 47, 12-19. http://dx.doi.org/10.1037/a0018849

Owen, I. R. (1992). Applying social constructionism to psychotherapy. Counselling Psychology Quarterly, 5, 385402. http://dx.doi.org/10.1080/09515079208254483

Parker, I., Georgaca, E., Harper, D., McLaughlin, T. y Stowell-Smith, M. (1995). Deconstructing psychopathology. Londres, Reino Unido: Sage.

Pérez Álvarez, M. y García Montes, J. M. (2007). The Charcot effect: The invention of mental illnesses. Journal of Constructivist Psychology, 20, 309-336. http://dx.doi.org/10.1080/10720530701503843

Pilgrim, D. (1997). Psychotherapy and society. Londres, Reino Unido: Sage.

Pope, K. S. y Vasquez, M. J. T. (2011). Ethics in psychotherapy and counselling (4a ed.). Nueva Jersey, NJ: John Wiley and Sons.

Prilleltensky, I. (1997). Values, assumptions, and practices. American Psychologist, 52, 517-535. http:// dx.doi.org/10.1037/0003-066X.52.5.517

Richardson, F. C. (2012). On psychology and virtue ethics. Journal of Theoretical and Philosophical Psychology, 32, 24-34. http://dx.doi.org/10.1037/a0026058

Robinson, D. N. (1976). An intellectual history of psychology. Madison, WI: The University of Wisconsin Press.

Robinson, D. N. (1997). Therapy as theory and civics. Theory \& Psychology, 7, 675-681. https://bit.ly/2FTL02w

Rodolfa, E., Bent, R., Eisman, E., Nelson, P., Rehm, L. y Ritchie, P. (2005). A cube model for competency development: Implication for psychology educators and regulators. Professional Psychology: Research and Practice, 36, 347-354. http://dx.doi.org/10.1037/0735-7028.36.4.347

Rogers, C. (1957). The necessary and sufficient conditions of therapeutic personality change. Journal of Consulting Psychology, 21, 95-103. http://dx.doi.org/10.1037/h0045357

Rychlak, J. F. (2003). The human image in postmodern America. Washington: APA.

Roth, A. y Fonagy, P. (1996). What works for whom? A critical review of psychotherapy research. Nueva York, NY: Guilford Press.

Segal, Z., Teasdale, J. D. y Williams, J. M. G. (2002). Mindfulness-based cognitive therapy for depression: A new approach to preventing relapse. Nueva York, NY: Guilford Press.

Segovia, S. (2017). Mindfulness: un camino de desarrollo personal. Bilbao, España: DDB.

Seligman, M. E. P. (1995). The effectiveness of psychotherapy. The Consumer Reports Study. American Psychologist, 50, 965-974. https://doi.org/10.1037/0003-066X.50.12.965.

Slife, B. D. y Williams, R. N. (1995). What's behind the research? Discovering hidden assumptions in the behavioral sciences. Thousand Oaks, CA: Sage Publications.

Smith, K. R. (2009). Psychotherapy as applied science or moral praxis: The limitations of empirically supported treatment. Journal of Theoretical and Philosophical Psychology, 29, 34-46. https://bit.ly/2LFr0UQ

Stiles, W. N., Honos-Webb, L. y Surko, M. (1998). Responsiveness in psychotherapy. Clinical Psychology \& Psychotherapy, 5, 439-458. http://dx.doi.org/10.1111/j.1468-2850.1998.tb00166.x

Stiles, W. B., Osatuke, K., Glick, M. J. y Mackay, H. C. (2004). Encounters between internal voices generate emotion: An elaboration of the assimilation model. En H. H. Hermans y G. Dimaggio (Eds.), The dialogical self in psychotherapy (pp. 91-107). Nueva York, NY: Brunner-Routledge.

Strong, T. y Sutherland, O. (2007). Conversational ethics in psychological dialogues: Discursive and collaborative considerations. Canadian Psychology, 48, 94-105. http://dx.doi.org/10.1037/cp2007011

Tjeltveit, A. C. (2004). The good, the bad, the obligatory, and the virtuous: The ethical contexts of psychotherapy. Journal of Psychotherapy Integration, 14, 149-167. http://dx.doi.org/10.1037/1053-0479.14.2.149

Tjeltveit, A. C. (2006). To what ends? Psychotherapy goals and outcomes, the good life, and the principle of beneficence. Psychotherapy, 43, 186-200. https://doi.org/10.1037/0033-3204.43.2.186

Van Broeck, N. y Lietaer, G. (2008). Psychology and psychotherapy in health care. A review of legal regulations in 17 European countries. European Psychologist, 13, 53-63. http://dx.doi.org/10.1027/1016-9040.13.1.53

Wampold, B. E. (1997). Methodological problems in identifying efficacious psychotherapies. Psychotherapy Research, 7, 21-43. http://dx.doi.org/10.1080/10503309712331331853

Wampold, B. E. e Imel, Z. E. (2015). The great psychotherapeutic debate. The evidence for what makes psychotherapy works. Nueva York, NY: Routledge.

Woolfolk, R. L. (1992). Hermeneutics, social constructionism and other items of intellectual fashion: Intimations for clinical science. Behavior Therapy, 23, 213-223. https://bit.ly/2Jp2h4I

Woolfolk, R. L. y Murphy, D. (2004). Axiological foundations of psychotherapy. Journal of Psychotherapy Integration, 14, 168-191. http://dx.doi.org/10.1037/1053-0479.14.2.168 
Woolfolk, R. L. y Richardson, F. C. (2008). Philosophy and psychotherapy. Journal of Psychotherapy Integration, 18, 25-39. http://dx.doi.org/10.1037/1053-0479.18.1.25

Zur, O. (2007). Boundaries in psychotherapy. Ethical and clinical explorations. Washington, DC: APA. 